\title{
PAC Identification of Electric-Nuclear-Quadrupole Interactions in Sm-Sesquioxides
}

\author{
M. Rentería*, L. A. Errico ${ }^{+}$, A. G. Bibiloni*, K. Freitag ${ }^{\mathrm{a}}$, and F. G. Requejo* \\ Departamento de Física, Facultad de Ciencias Exactas, Universidad Nacional de La Plata, \\ C.C. 67,1900 La Plata, Argentina \\ ${ }^{\text {a }}$ Institut für Strahlen-und Kernphysik (ISKP) der Universität Bonn, \\ Nussallée 14-16, D-53115 Bonn \\ ${ }^{*}$ Member of CONICET; ${ }^{+}$fellow of CONICET, Argentina
}

Reprint requests to Dr. M. R.; Fax: 54-221-4252006; E-mail: renteria@ venus.fisica.unlp.edu.ar

Z. Naturforsch. 55 a, 155-159 (2000); received August 26, 1999

\begin{abstract}
Presented at the XVth International Symposium on Nuclear Quadrupole Interactions, Leipzig, Germany, July 25 - 30, 1999.
\end{abstract}

\begin{abstract}
We present Perturbed Angular Correlation (PAC) results of the electric-nuclear quadrupole interaction at ${ }^{181} \mathrm{Ta}$ located at the nonequivalent cation sites of cubic $\mathrm{C}-\mathrm{Sm}_{2} \mathrm{O}_{3}$ and monoclinic B-Sm $\mathrm{O}_{3}$ free of defects. The application of the empirical $\mathrm{Cd} / \mathrm{Ta}$ correlation found in binary oxides allowed us to correlate the interactions with each site and to identify the single Sm site populated in ${ }^{111}$ In-implanted $\mathrm{B}-\mathrm{Sm}_{2} \mathrm{O}_{3}$ experiments. The preferential site occupation of Cd and Ta in B-Samaria is discussed in terms of the "chemistry" of the probe-atoms.
\end{abstract}

Key words: Perturbed-Angular Correlations; Electric-Field Gradients; $\mathrm{Sm}_{2} \mathrm{O}_{3}$; Rare-earth Semiconductor and Insulator Oxides; ${ }^{181} \mathrm{Hf}$-Impurity Implantation.

\section{Introduction}

A systematic study of the electric-field gradient (EFG) at ${ }^{111} \mathrm{Cd} /{ }^{181} \mathrm{Ta}$ impurity sites in binary oxides has been carried out during the last ten years by means of the Perturbed-Angular-Correlation (PAC) technique investigating the EFG dependence on the probe-atom electronic configuration and on the coordination with its nearest neighbours $(\mathrm{NN})$ in the host [1 - 3]. Recently we reported that the empirical correlation between local (valence) and ionic contributions to the EFG found for ${ }^{111} \mathrm{Cd}[1]$ depends on the electronic configuration of the probe and a simple EFG factorization was proposed for bixbyites [4], C$\mathrm{Sm}_{2} \mathrm{O}_{3}$ among them:

$$
\begin{aligned}
& V_{z z}^{\text {Exp }}=V_{z z}^{\text {Local }}+V_{z z}^{\text {Ionic }} \\
& \quad=\left(1-\mu_{\mathrm{NN}}^{\prime}\right)^{\text {site } i \text {, probe } i} V_{z z}^{\text {Ionic }} \\
& \quad=\left(1-\mu_{\mathrm{NN}}^{\prime}\right)^{\text {site } i \text {, probe } i}\left(1-\gamma_{\infty}\right)^{\text {probe } i} V_{z z}^{\text {latt }}(\text { site } i) .
\end{aligned}
$$

Some years ago Lupascu et al. [5] reported first PAC experiments on ${ }^{111}$ In-implanted Sm-, Gd-, and $\mathrm{Eu}-$ sesquioxides in their high-temperature monoclinic modification (B-phase), which has 3 nonequivalent cation sites, but they only observed one EFG in each oxide. Our aim was to extend our comparative study to this group, given that it presents 3 additional coordination geometries and a rather small unit cell that make feasible future FP-LAPW EFG calculations at impurity sites [6]. In this work we present PAC results of NQI at ${ }^{181} \mathrm{Ta}$ located at all cation sites of $\mathrm{C}-\mathrm{Sm}_{2} \mathrm{O}_{3}$ and $\mathrm{B}-\mathrm{Sm}_{2} \mathrm{O}_{3}$.

\section{Experimental}

\section{Crystal Structures}

$\mathrm{Sm}_{2} \mathrm{O}_{3}$ crystallises mainly in two forms in a wide temperature range [7]: the cubic bixbyite structure (Cphase, class Ia3), stable below $T_{\mathrm{c}}=1123 \mathrm{~K}$, presents two 6-fold oxygen-coordinated (M-O) cation sites "C" (highly asymmetric) and " $D$ " (axially symmetric) with 3:1 abundance. This phase transforms at $T_{\mathrm{c}}$ to the monoclinic B-phase (class $\mathrm{C} 2 / \mathrm{m}$ ) that presents three nonequivalent 7 -fold coordinated cation sites "Sm1", "Sm2", and "Sm3" equally abundant in the 

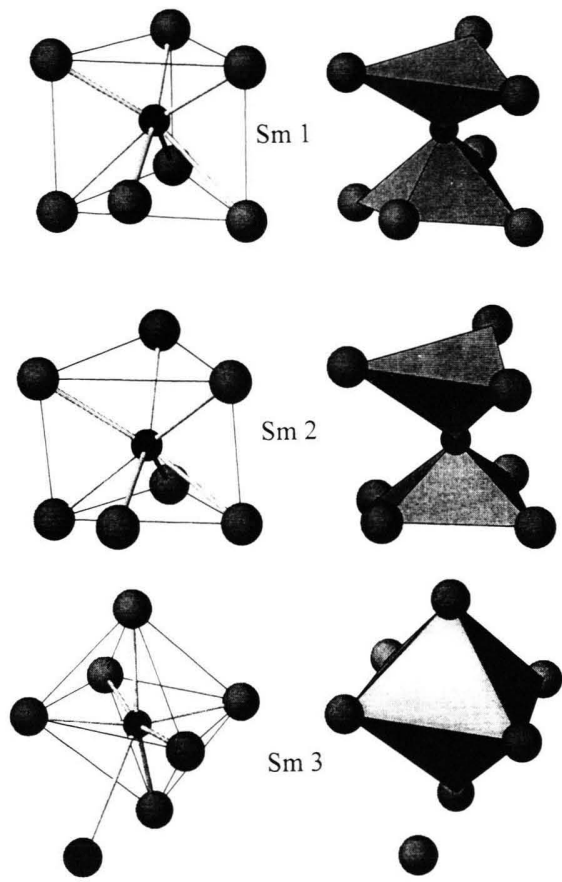

Fig. 1. Ball and stick (left) and polyhedra (right) drawings showing the nonequivalent coordination of Sm (black balls) with $\mathrm{NN}$ oxygen ions (grey balls) in $\mathrm{B}-\mathrm{Sm}_{2} \mathrm{O}_{3}$.

lattice [8] (see Figure 1). In Sm1 and Sm2 six oxygen atoms form a trigonal prism with the seventh oxygen further away. In Sm3, the seventh oxygen is so far away (3.12 $\AA$, compared to a mean of $2.38 \AA$ of the six others) that the rest can be seen as an octahedron.

\section{Sample Preparation and Measurements}

From commercially-obtained high-purity $\mathrm{Sm}_{2} \mathrm{O}_{3}$ powder $(99.999 \%)$ two pellets were pressed, one treated at $773 \mathrm{~K}$ for $27 \mathrm{~h}$ (sample I) and the other treated at $1303 \mathrm{~K}$ for $48 \mathrm{~h}$ (sample II). XRD analysis revealed that the powder is much disordered with some peaks of B-phase, sample I consist mostly of crystalline C-phase with 15 - 20\% B-phase, and sample II consist of pure crystalline B-phase. ${ }^{181} \mathrm{Hf}^{+}$ $\left(\beta^{-} \rightarrow{ }^{181} \mathrm{Ta}\right)$ ions were implanted into the samples by means of the ion accelerator of the ISKP (Bonn) with energies of $160 \mathrm{keV}$ and doses of $1.5 \times 10^{13}$ ions $/ \mathrm{cm}^{2}$. The PAC measurements were made in air at 290 $1373 \mathrm{~K}$ after the annealing treatments indicated in Fig. 2 (right). The $133-482 \mathrm{keV} \gamma-\gamma$ cascade of ${ }^{181} \mathrm{Ta}$ was observed with a conventional four-CsF-detectors PAC set-up with fast-fast logic (time resolution of
$0.9 \mathrm{~ns})$. A brief description of the used data reduction can be found in [9].

\section{Results}

The PAC and Fourier spectra of sample I (see Fig. 2) showed essentially no change below $T_{\mathrm{c}}$ $(=1123 \mathrm{~K})$ but a drastic change after the annealing above $T_{\mathrm{c}}$, in agreement with a total phase transformation monitored by parallel XRD measurements. Five well-defined $(\delta<2 \%)$ hyperfine interactions (Hfi) were found to be nearly constant in the whole temperature range. The constancy of the parameters of the Hfi's C, D, and B2, fitted below $T_{\mathrm{c}}$, is shown in Figure 3. The Hfi's $\mathrm{C}$ and $\mathrm{D}$ were already assigned to defect-free cation sites $\mathrm{C}$ and $\mathrm{D}$ in C-Sm${ }_{2} \mathrm{O}_{3}$ [4] based on the agreement with the relative populations predicted, EFG symmetry and strength ratio, and with the systematic in bixbytes [3]. Three monochromatic Hfi's B1, B2, and B3 were found above $T_{\mathrm{c}}$ with nonequivalent populations identical within errors to those found in sample II (see Table 1). This agreement with sample II reinforces the previous assignment of the Hfi's C and D, given that Hfi B2 (below $T_{\mathrm{c}}$ ) obviously belongs to the B-phase.

\section{Discussion and Conclusions}

The Hfi's B1, B2 and B3 in sample I are assigned to probes in the three defect-free cation sites of BSamaria based on: they are well defined and constant at $290-1373 \mathrm{~K}$ (see Fig. 4) and identical to the Hfi's found in sample II. The comparison of the $\eta$ values with predictions of the point-charge model (PCM) [2] in Table 1, although similar for B2 and B3, can hardly be used as is usual in ${ }^{111} \mathrm{Cd}$ experiments, since it frequently fails at ${ }^{181} \mathrm{Ta}$ in oxides [9]. Based on the strengths of B2 and B3 they can not be related with sites Sm1 since it has a smaller EFG PCM prediction (see Table 1). Hence, we attribute B1 to Sm1. The most populated fraction $\mathrm{B} 2$ is the one that exists below $T_{\mathrm{c}}$, showing a preferential Ta occupancy of one site. On the other hand, B3 is least populated. This fact, not found for Cd-probes until the experiment reported by Lupascu et al. [5], was already detected for ${ }^{181} \mathrm{Ta}$ in bixbyites, depending on the relative ionic size of the probe and the cationic "space" of the host, as well as on how different the NN cation coordination is from that of $\mathrm{Hf}$ in its own oxide, $\mathrm{m}-\mathrm{HfO}_{2}$ [3]. Site $\mathrm{Sm} 2$ has a coordination very similar to that of $\mathrm{m}$-Hafnia, while 


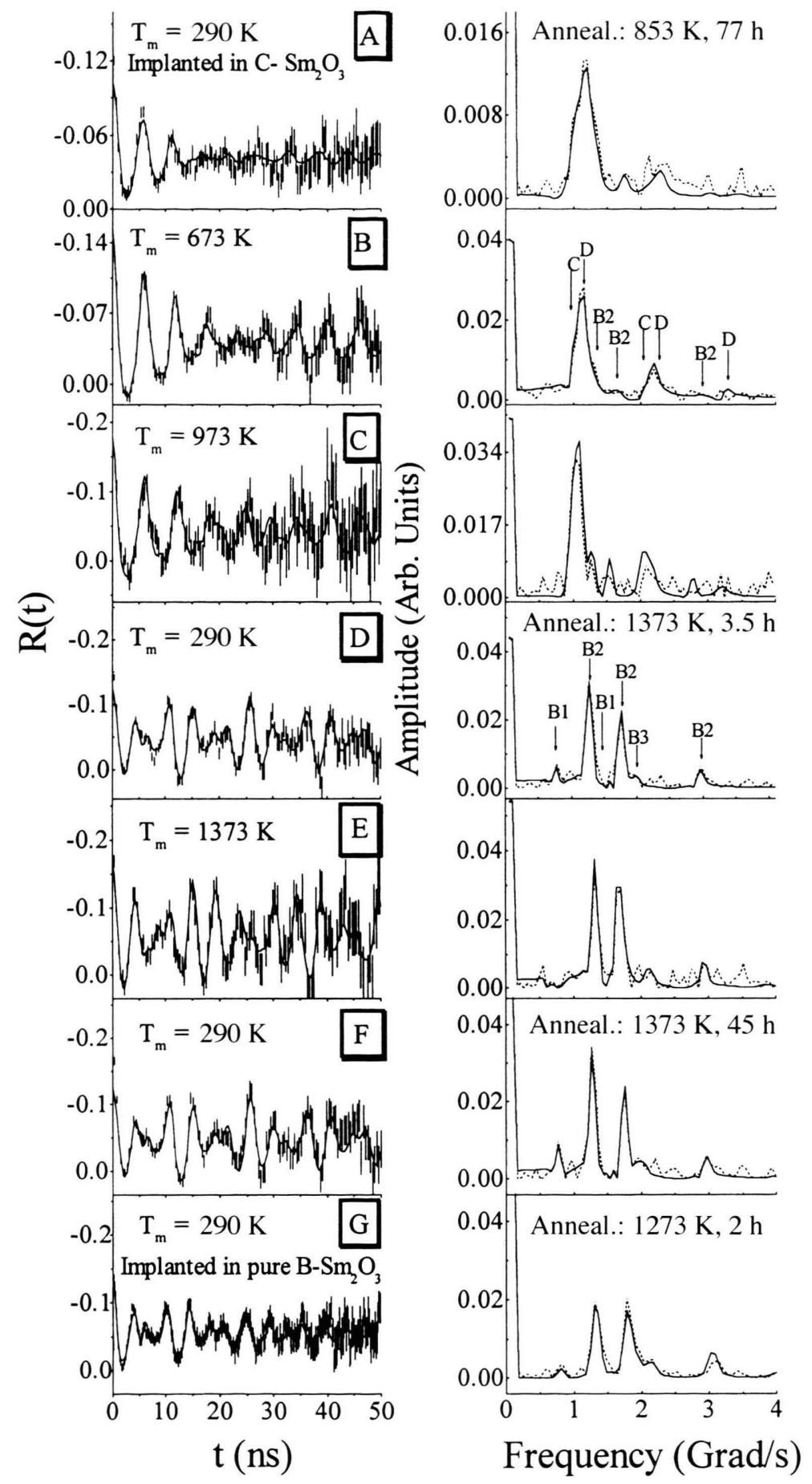

Fig. 2. PAC (left) and Fourier (right) spectra of ${ }^{181} \mathrm{Hf}$-implanted into sample I (A-F) and sample II $(G)$ at indicated measuring temperatures. The solid line is a leastsquares fit of usual static perturbation factors to the data [9]. The interaction frequencies $\omega_{i}$ of all observed interactions are marked on the Fourier spectra.

Sm3 has an octahedral M-O coordination and mean distances similar to In sites in the bixbyite structure of $\mathrm{In}_{2} \mathrm{O}_{3}$ (see Figure 1). Hence, based on the chemistry of the probe-atom, we tentatively assign B2 and B3 to $\mathrm{Sm} 2$ and $\mathrm{Sm} 3$, respectively. We checked these assumptions by applying the ${ }^{181} \mathrm{Ta}$ empirical correlation 
Table 1. Hyperfine parameters of interactions B1, B2, and B3 found in sample I after its phase transformation into the B-phase. The first and second row correspond to the spectra $(F)$ and $(E)$ of Fig. 2, respectively. Results are compared with predictions within the point - charge model (PCM) and those coming from the application of the ${ }^{181} \mathrm{Ta}$ empirical correlation (see text), and with PAC results obtained from sample II (implantation in pure B-Sm $\mathrm{O}_{3}$, last row).

\begin{tabular}{|c|c|c|c|c|c|c|c|c|c|}
\hline \multirow[b]{2}{*}{ Probe: ${ }^{181} \mathrm{Ta}$} & \multirow[b]{2}{*}{ Hfi's } & \multicolumn{4}{|c|}{ - PAC Results - } & \multirow[b]{2}{*}{ Sm Site } & \multicolumn{2}{|c|}{ - Calculations - } & \multirow[b]{2}{*}{$\eta(\mathrm{PCM})$} \\
\hline & & $\begin{array}{l}\text { Fraction } \\
(\%)\end{array}$ & $\begin{array}{l}\omega_{\mathrm{Q}} \\
(\mathrm{Mrad} / \mathrm{s})\end{array}$ & $\eta$ & $\begin{array}{l}\delta \\
(\%)\end{array}$ & & $\begin{array}{l}\omega_{\mathrm{Q}}^{\text {Correlations }} \\
(\mathrm{Mrad} / \mathrm{s})\end{array}$ & $\begin{array}{l}\omega_{\mathrm{Q}}^{\text {Ifnic, PCM }} \\
(\mathrm{Mrad} / \mathrm{s})\end{array}$ & \\
\hline$T_{m}=290 \mathrm{~K}$ & B1 & $13(2)$ & $68.6(6)$ & $\underline{1}$ & $2(1)$ & Sml & $-41(1)$ & $26.2(9)$ & $0.1(2)$ \\
\hline \multirow[t]{2}{*}{ Sample I } & B2 & $79(3)$ & $152.7(3)$ & $\overline{0} .619(3)$ & $0.7(2)$ & $\mathrm{Sm} 2$ & $-108.7(4)$ & $71.9(3)$ & $0.42(4)$ \\
\hline & B3 & $8(1)$ & $190(2)$ & $0.94(2)$ & $0.5(5)$ & Sm3 & $-190.2(8)$ & $134.5(6)$ & $0.82(1)$ \\
\hline$T_{m}=1373 \mathrm{~K}$ & B1 & $6(1)$ & 69 (2) & $0.712(4)$ & $0.3(2)$ & - & - & - & - \\
\hline \multirow{2}{*}{ Sample I } & B2 & $71(6)$ & $148.1(3)$ & $0.78(2)$ & $2(1)$ & - & - & - & - \\
\hline & B3 & $23(6)$ & $191(2)$ & $0.73(6)$ & $1(1)$ & - & - & - & - \\
\hline$T_{m}=290 \mathrm{~K}$ & B1 & $10(2)$ & $71(1)$ & $\underline{1}$ & $5(2)$ & - & - & - & - \\
\hline \multirow[t]{2}{*}{ Sample II } & B2 & $70(3)$ & $156.2(3)$ & $0.631(3)$ & $2.2(2)$ & - & - & - & - \\
\hline & B3 & $20(3)$ & $194(3)$ & $0.87(2)$ & $5(2)$ & - & - & - & - \\
\hline
\end{tabular}

Table 2. Comparison between the single hyperfine interaction found for ${ }^{111}$ In-implanted B-Samaria [5] and predictions within the PCM and the ${ }^{111} \mathrm{Cd}$ empirical correlation for the three cationic sites (see text).

\begin{tabular}{cllllllll}
\hline Probe: ${ }^{111} \mathrm{Cd}$ & Hfi's & Fraction $(\%)$ & $\omega_{\mathrm{Q}}^{\text {Exp }}(\mathrm{Mrad} / \mathrm{s})$ & $\eta$ & Sm Site & $\omega_{\mathrm{Q}}^{\text {Correlation }}(\mathrm{Mrad} / \mathrm{s})$ & $\omega_{\mathrm{Q}}^{\text {Ionic, (PCM) }}(\mathrm{Mrad} / \mathrm{s})$ & $\eta^{(\mathrm{PCM})}$ \\
\hline$T_{m}=290 \mathrm{~K}$ & $\mathrm{~B} 1$ & - & - & - & $\mathrm{Sm} 1$ & $-7.0(6)$ & $4.5(4)$ & $0.1(2)$ \\
& $\mathrm{B} 2$ & - & - & - & $\mathrm{Sm} 2$ & $-19.1(5)$ & $12.3(3)$ & $0.42(4)$ \\
& $\mathrm{B} 3$ & 100 & $32.51(5)$ & $0.979(5)$ & $\mathrm{Sm} 3$ & $-35.6(5)$ & $22.9(3)$ & $0.82(1)$ \\
\hline
\end{tabular}

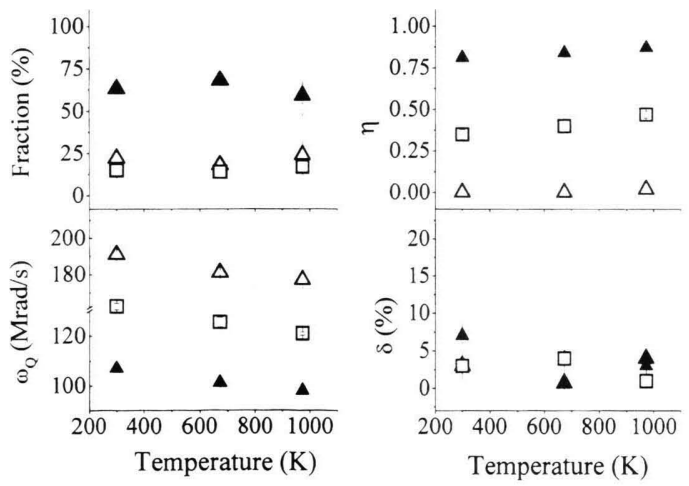

Fig. 3. Evolution of the hyperfine parameters before the phase transition (sample I) as functions of measuring temperature: Hfi D: $\Delta$, Hfi C: $\mathbf{\Lambda}, \mathrm{Hfi}$ B2: $\square$.

to this structure (see Figure 5). The points for Sm 1, 2, and 3 were calculated assuming that the Hfis's B1, B2, and B3 correspond to probes in Sm-sites Sm1, Sm2, and Sm3 (solid circles in Fig. 5) and that the Hfi's B1, B2, and B3 corresponds to Sm 1, Sm3, and Sm2 (open squares), respectively. Figure 5 shows that the first assumption (solid circles) is much better. Table 1 also shows the excellent agreement between the experimental quadrupole frequencies and those predicted

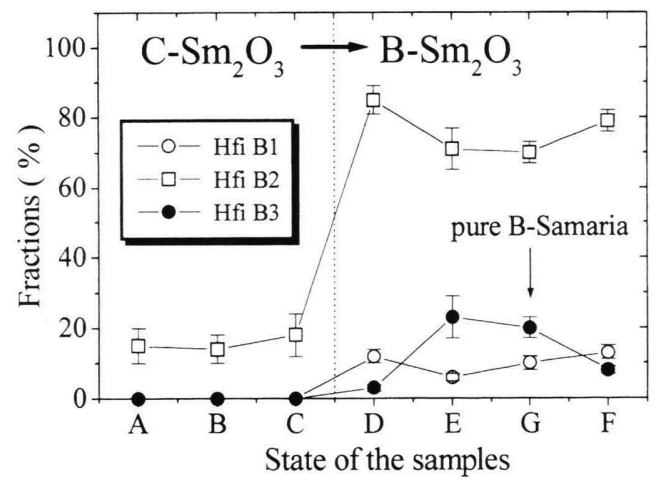

Fig. 4. Evolution of fractions B1, B2, and B3 in sample I before (A-C) and after (D-F) the phase transformation. Data $\mathrm{G}$ correspond to Hfi's in sample II.

by the correlation (dashed line in Fig. 5) using a general value of the $\mu$-factor in (1) of $2.5[1,4]$ extracted from the systematics. The increase of the least populated fraction B3 at high temperature (see Fig. 4) must then be correlated with the onset of ionic conductivity above $800 \mathrm{~K}$ [5] that allows $10 \%$ of the Ta ions to jump into the other two cation sites, in agreement with the reversible character of this effect (fraction B3 diminishes again at $290 \mathrm{~K}$ in spectrum F, see Figure 4). The 


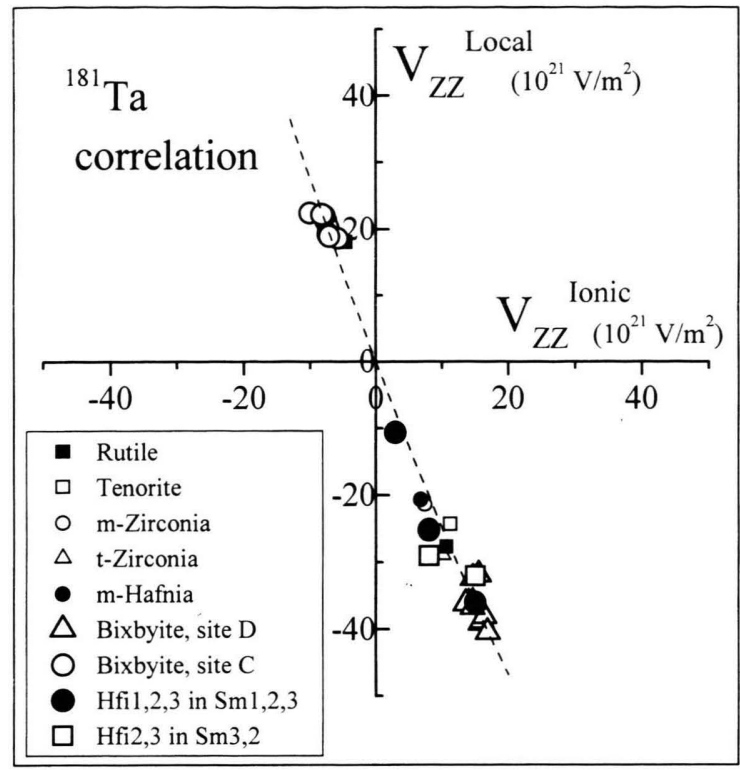

Fig. 5. Empirical correlation found for ${ }^{181}$ Ta in binary oxides $[1,4]$. The dashed line is a polynomial fit to the data. Arrows indicate $V_{Z Z}{ }^{\text {Ionic }}$ for $\mathrm{Sm} 1, \mathrm{Sm} 2$, and $\mathrm{Sm} 3$.

implantation process in pure B-Samaria (spectrum G, Fig. 4) that localises the Hf ions at cation sites using their kinetic energy seems to play the same role as keeping the temperature high. The same analysis as done before with the correlation enables to identify the single Hfi seen in ${ }^{111} \mathrm{Cd}$ experiments. Figure 6 shows that the population of $\mathrm{Sm} 3$ gives the better agreement with the ${ }^{111} \mathrm{Cd}$ correlation, in agreement with the assignment done by the Göttingen group [5]. Finally, Table 2 shows the excellent agreement obtained from the correlation using the $\mu$-factor found for $\mathrm{ZrO}_{2}\left({ }^{111} \mathrm{Cd}\right)$ [10] since it presents a 7-fold coordination like Sm3. PAC studies on ${ }^{181} \mathrm{Hf}$-implanted

[1] M. Rentería, C. P. Massolo, and A. G. Bibiloni., Mod. Phys. Lett. B6, 1819 (1992); R. Weht, G. Fabricius, M. Weissmann, M. Rentería, C. P. Massolo, and A. G. Bibiloni, Phys. Rev. B49, 14939 (1994).

[2] D. Wiarda, M. Uhrmacher, A. Bartos, and K. P. Lieb, J. Phys.: Condens. Matter 5, 4111 (1992) and references therein.

[3] M. Rentería, A. G. Bibiloni, F. G. Requejo, A. F. Pasquevich, J. Shitu, L. A. Errico, and K. Freitag, Mod. Phys. Lett. B12, 819 (1998).

[4] M. Rentería, K. Freitag, and L. A. Errico, Hyp. Int. 120/121, 449 (1999).

[5] D. Lupascu, M. Uhrmacher, and K. P. Lieb, J. Phys.: Condens. Matter 6, 10445 (1994).

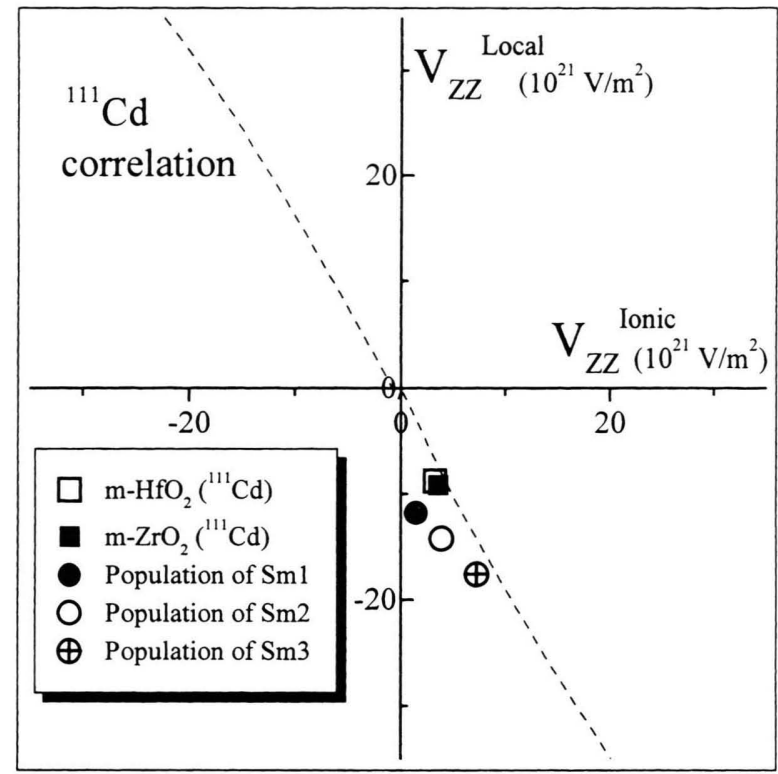

Fig. 6. Empirical correlation found for ${ }^{111} \mathrm{Cd}$ in binary oxides [1]. The dashed line is a polynomial fit to the data, not shown for simplicity. Circles represent the prediction of $V_{Z Z}{ }^{\text {Local }}$ if each Sm site should be the one populated in ${ }^{111} \mathrm{Cd}$ experiments [5].

C- and B-phase Gd- and Eu-sesquioxides and corresponding ab initio EFG calculations at impurity sites, already in progress, will certainly be of great value for the understanding of the electric-nuclear quadrupole interaction in these systems.

This work was partially supported by CONICET, Fundación Antorchas, CICpBA and ANPCyT (PICT98 03 - 03727), Argentine, and The Third World Academy of Sciences (TWAS), Italy, RGA 97-057. The neutron irradiation performed by the GKSS reactor FRG-1, Germany, is kindly acknowledged.

[6] L. A. Errico, G. Fabricius, and M. Rentería, Z. Naturforsch. A, (1999), in press.

[7] L. Eyring, Handbook on the Physics and Chemistry of the Rare Earths, ed. K. A. Gschneidner and L. Eyring, North Holand 1979, p. 337.

[8] T. Schleid and G. Meyer, J. Less-Comm. Met. 149, 73 (1989).

[9] M. Rentería, F. G. Requejo, A. G. Bibiloni, A. F. Pasquevich, J. Shitu, and K. Freitag, Phys. Rev. B55, 14200 (1997).

[10] J. Luthin, K. P. Lieb, N. Neubauer, and M. Uhrmacher, Phys. Rev. B57, 15272 (1998). 